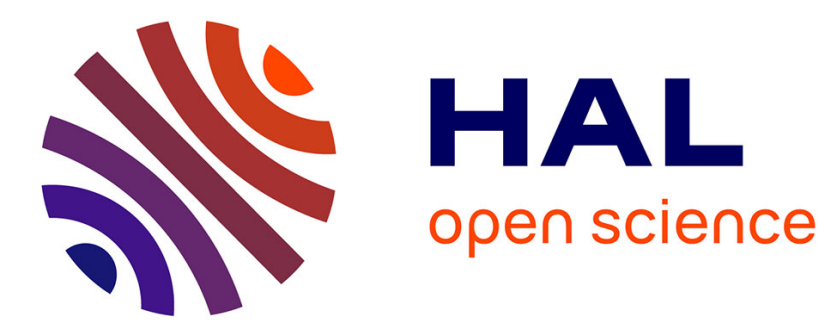

\title{
A novel approach for studying the indoor dispersion of aroma through computational fluid dynamics
}

Artemio Plana-Fattori, Ioan-Cristian Trelea, Jean-Francois Le Page, Isabelle

Souchon, Philippe Pollien, Santo Ali, Marco Ramaioli, Estelle

Pionnier-Pineau, Christoph Hartmann, Denis Flick

\section{To cite this version:}

Artemio Plana-Fattori, Ioan-Cristian Trelea, Jean-Francois Le Page, Isabelle Souchon, Philippe Pollien, et al.. A novel approach for studying the indoor dispersion of aroma through computational fluid dynamics. Flavour and Fragrance Journal, 2014, 29 (3), pp.143-156. 10.1002/ffj.3190 . hal-01123063

\section{HAL Id: hal-01123063 \\ https://hal.science/hal-01123063}

Submitted on 11 Jul 2017

HAL is a multi-disciplinary open access archive for the deposit and dissemination of scientific research documents, whether they are published or not. The documents may come from teaching and research institutions in France or abroad, or from public or private research centers.
L'archive ouverte pluridisciplinaire HAL, est destinée au dépôt et à la diffusion de documents scientifiques de niveau recherche, publiés ou non, émanant des établissements d'enseignement et de recherche français ou étrangers, des laboratoires publics ou privés. 
Full title:

\section{A Novel Approach for Studying the Indoor Dispersion of Aroma through Computational Fluid Dynamics}

Short title (70 characters):

\section{Aroma Indoor Dispersion through Computational Fluid Dynamics}
A. Plana-Fattori ${ }^{(1,2,3)}$, I. C. Trelea ${ }^{(4,5)}$, J. F. Le Page ${ }^{(1,2,3)}$, I. Souchon ${ }^{(4,5)}$, P. Pollien ${ }^{(6)}$, S. Ali ${ }^{(6)}$, M. Ramaioli ${ }^{(6)}$, E. Pionnier-Pineau ${ }^{(6)}$, C. Hartmann ${ }^{(6)}$, and D. Flick ${ }^{(1,2,3)}$

(1) AgroParisTech, UMR1145 Ingénierie Procédés Aliments, F-75005 Paris, France

(2) INRA, UMR1145 Ingénierie Procédés Aliments, F-91300 Massy, France

(3) Le CNAM, UMR1145 Ingénierie Procédés Aliments, F-75003 Paris, France

(4) AgroParisTech, UMR782 Génie et Microbiologie des Procédés Alimentaires, F-78850 Thiverval-Grignon, France

(5) INRA, UMR782 Génie et Microbiologie des Procédés Alimentaires, F-78850 ThivervalGrignon, France

(6) Nestlé Research Center, CH-1000 Lausanne, Switzerland

Corresponding Author Address:

Artemio Plana-Fattori

AgroParisTech - MMIP, 16 rue Claude Bernard, 75231 Paris CEDEX 5, France

phone: +33.1.44.08.86.84 ; fax: +33.1 .44 .08 .16 .66$

e-mail: artemio.planafattori@agroparistech.fr 


\section{Abstract}

We propose a mechanistic modeling approach for studying the indoor dispersion of aroma compounds which are released from, for instance, food products. The approach combines the indoor velocity field with a release model for aroma compounds. The release mass flux is expressed as a function of key variables such as mass transfer and gas-liquid partition coefficients, and the source geometry. The transport properties of ambient air are assumed to be independent of the aroma concentration; hence release and dispersion problems can be solved separately. Firstly, the velocity field is obtained as solution of the fluid flow problem through computational fluid dynamics (CFD). The turbulent velocity field is then used to predict the time evolution of concentration of an aroma compound released by a constant rate source, in an initially aroma-free environment. These results are interpreted in terms of a step response function. The aroma concentration as a function of time is finally estimated by convolving the possibly time-varying release mass flux and the response function associated with the position of interest. The modeling approach is flexible and computationally effective, since different release models as well as the release of distinct aroma compounds can be directly studied by taking into account a same velocity field, without any additional CFD simulation. The validity of the approach is assessed from measurements of aroma concentration in a $140 \mathrm{~m}^{3}$ room, under constant release mass flux. The approach is also illustrated for a case where the release mass flux is not constant in time.

\section{Brief Abstract (for graphical Flavour and Fragrance Journal's table of contents)}

We propose a modeling approach for studying the indoor dispersion of aroma compounds which are released from, for instance, food products. We show that the indoor velocity field can be estimated separately through CFD, under aroma-free conditions, and then combined with the aroma mass flow associated with the source of interest. Modeling results compare favorably with experimental measurements. The modeling approach is flexible and computationally effective, since different release conditions can be considered with no additional CFD simulation.

\section{Keywords}

aroma release model, indoor dispersion, computational fluid dynamics (CFD), turbulent flow, PTR-MS 


\section{Introduction}

Consumer behavior is a widely studied subject in food science and technology. Different spatial and temporal scales can be involved, since the first perception of the food product by the consumer with the help of her / his smell and vision, up to the direct interaction after the food product reaches the mouth. Efforts have been accomplished in understanding the mechanisms which drive the release and the perception of flavor under in vivo conditions [1-5]. In this context, detailed mechanistic models for representing the aroma release have been developed, providing useful tools for identifying the most important physicochemical, anatomical, and physiological parameters which drive this release [6].

The air flow which prevails around the food product and the consumer can influence the orthonasal olfactory perception and potentially his consumption experience and behavior [7]. The influence of ventilation conditions on the dispersion of aroma compounds released by food matrices follows the physical laws which drive the dispersion of any gaseous species and also of small particles suspended in the air. In the case of indoor environments, perfectlymixed conditions have been often assumed in characterizing indoor physical phenomena. Such an assumption proves too simplistic for the initial period of indoor dispersion; furthermore, experimental work has shown that rooms may not become well-mixed over any length of time [8].

The dispersion of gaseous species and particles in the air has been studied mainly because of their potential impact on human health and climate [9]. The subject has progressed since the theory of atmospheric dispersion in the 1940's [10] up to numerical models which can predict the air flow and the particle concentration fields throughout relatively large environments like an urban canyon [11]. The progressive increase of computer resources along past decades has widened the field of application of the computational fluid dynamics (CFD), including the ventilation and air indoor science. However, much previous work applying CFD simulations to the indoor dispersion has been performed without corresponding experimental work [8], while the combination of experiment and modeling work appears as the effective scientific approach for ventilation studies [12-16].

Realistic modeling of aroma dispersion from the food product to the consumer requires, as a key input, information about the release of aroma from the food matrix (that is, 
about its emission by the food matrix). A similar problem which has been widely studied is the release of volatile and semi-volatile organic compounds, (S)VOCs. These compounds can be emitted by a number of commonly used dry materials such as wood products, floor coverings (carpet, vinyl), wall coverings (wallpaper, fabric), ceiling materials (acoustic tiles, subfloors), and insulation materials (fiberglass, rigid foam) [17]. Architectural coatings applied to building materials can also act as sources of (S)VOCs, either during the painting process (through evaporation) or after the paint application (through progressive release from the substrate) [18]. (S)VOCs emissions are important to air quality because of their large surface area and permanent exposure to indoor air. The potential impact of these compounds on health [19] has justified the measurement of their concentration as well as the modeling of their emission. The emission of (S)VOCs due to many materials under indoor and outdoor conditions has been modeled through a number of strategies; the most relevant ones have been compiled and their validity and usefulness have been discussed [20-22].

In this exploratory study, we propose and test a modeling approach for representing aroma release and dispersion throughout an indoor environment under forced ventilation. Plausible assumptions are made: for instance, the indoor environment is assumed to remain well below the saturation level with respect to the aroma compounds of interest; hence, the release of aroma depends only on the concentration close to the product surface. The release model can be seen as an application of the interfacial mass transfer theory for studying the volatile release from aqueous solutions [4, 23]. The dispersion model uses the indoor velocity field, which is estimated through CFD simulations.

The article is organized as follows. The modeling approach of aroma release and dispersion is presented in Section 2. The approach is illustrated with the help of a case study built around an indoor environment which effectively exists (a test room), whereby measurements of air velocity and aroma concentration have been performed. Section 3 characterizes the test room, describes the experimental setup, and overviews the numerical model which is employed for estimating the indoor velocity field. Results discussed in Section 4 include a) the air flow and aroma concentration fields under steady-state conditions, b) comparisons of air velocities from model and experiment at selected positions, and c) the time evolution and the spatial distribution associated with the aroma concentration from model and experiment. Section 4 includes also results for a number of cases in which the aroma release depends on the time. Conclusions are summarized in Section 5. 


\section{Modeling approach}

The study of aroma dispersion requires information regarding key variables associated with the indoor environment itself: room geometry, incoming air flow rate, inlet and outlet position(s), source position(s). A second set of key variables is associated with the aroma release: source geometry, source temperature and parameters driving the release mass flow, and of course their dependence with time.

Our modeling approach includes two steps, hereafter referred as the release model and the dispersion model. Subsection 2.1 expresses mass flow rates at a gas-liquid interface, then discusses the validity of the assumptions, and concludes presenting a time-dependent release function. Subsection 2.2 discusses the possible simplifications of such a general approach in the case of aroma release from pure liquid compounds. Subsection 2.3 introduces the step response function, which provides the time evolution of the concentration associated with an aroma compound in an initially aroma-free condition, and finally describes the application of such a function in estimating the aroma concentration as function of position and time.

\subsection{Aroma release model}

In the case of an aroma compound diluted in a solution in contact with ambient air, the mass transfer resistance is assumed to be concentrated in two thin boundary layers around the interface between the liquid and gas phases [4, 23, 24]. Away from the boundary layer, the bulk aroma concentration is assumed to be spatially uniform, although it can vary in time. On the liquid side of the interface, the mass flux density $j_{l}\left(\mathrm{~kg} / \mathrm{m}^{2} / \mathrm{s}\right)$ across the liquid boundary layer can be expressed as:

$$
j_{l}=k_{l}\left(C_{l}-C_{l}^{*}\right)
$$

where $k_{l}$ is the mass transfer coefficient in the liquid $(\mathrm{m} / \mathrm{s})$, and $C_{l}$ and $C_{l}^{*}$ are the concentration values $\left(\mathrm{kg} / \mathrm{m}^{3}\right)$ in the liquid bulk and at the liquid-gas interface, respectively. Similarly, on the gas side of the interface,

$$
j_{g}=k_{g}\left(C_{g}^{*}-C_{g}\right)
$$

Under local thermodynamic equilibrium, the gas / liquid partition coefficient at the interface can be expressed as $K_{g l}=C_{g}^{*} / C_{l}^{*}$. The partition coefficient $K_{g l}$ strongly depends on the 
temperature and on the composition of the liquid product. Assuming no aroma accumulation at the interface, mass flux densities on liquid and gas sides are equal $\left(j_{l}=j_{g}=j\right)$. The mass density flux $j$ across the liquid-gas interface can be expressed in terms of the bulk concentrations $C_{l}$ and $C_{g}$ only, after taking the gas phase as reference:

$$
j=k_{t}\left(K_{g l} C_{l}-C_{g}\right)
$$

with

$$
\frac{1}{k_{t}}=\frac{1}{k_{g}}+\frac{K_{g l}}{k_{l}} .
$$

Equations (3) and (4) form the aroma volatile release model for diluted solutions. Equation (3) shows that the macroscopic driving force for the transfer is the "corrected" concentration difference $K_{g l} C_{l}-C_{g}$. The correction concerns the relative preference of the aroma molecules for the liquid phase, which is expressed via the partition coefficient. Equation (4) is a "resistance in series" formula for the total mass transfer $k_{t}$ coefficient, with a correction for the discontinuity in the concentration profile at the interface. Given the typical orders of magnitude assumed by its parameters $\left(k_{g} \sim 10^{-3} \ldots 10^{-2} \mathrm{~m} / \mathrm{s}, K_{g l} \sim 10^{-4} \ldots 10^{-2}, k_{l} \sim\right.$ $10^{-8} \ldots 10^{-6} \mathrm{~m} / \mathrm{s}$ ), the resistance on the liquid side of the interface is usually dominant.

Equation (3) expresses the total mass flux density across the liquid-gas interface $(j)$ as a function of the bulk concentration in the gas phase $\left(C_{g}\right)$. One difficulty to apply equation (3) comes from the fact that $C_{g}$ can be effectively known only after (and not before) solving the aroma dispersion problem throughout the indoor environment. The solution of this problem requires the availability of the mass flux across the interface itself. In order to decouple the dependence between $j$ and $C_{g}$, we hereafter assume that the system will always remain far from the bulk concentration equilibrium:

$$
C_{g}<<K_{g l} C_{l}
$$

In such a case, the driving force is independent on the bulk concentration in the gas phase. In addition, we assume that the low aroma concentrations in the air do not influence significantly its physical properties (namely: density and dynamic viscosity) which allows computing mass 
exchange coefficients considering pure air physical properties. As a result, the flux of aroma released by the source is independent on the aroma dispersion throughout the room.

The concentration of aroma compound in the source solution decreases with time as the release progresses. The mass balance of the aroma compound in the liquid phase can be expressed as:

$$
V_{l} \frac{d C_{l}}{d t}=-j A_{l}
$$

where $V_{l}$ is the volume of the liquid phase and $A_{l}$ the cross-sectional release area. Taking account of equation (3) and assuming the validity of condition (5), the time evolution of the aroma compound concentration in the liquid source is given by:

$$
\frac{d C_{l}}{d t}=-\frac{k_{t} K_{g l} A_{l}}{V_{l}} C_{l}
$$

Under the previous assumptions, the solution of this equation is straightforward, and it can be presented as:

$$
\begin{aligned}
& C_{l}\{t\}=C_{l}\{t=0\} \exp \left(-t / \tau_{l}\right) \\
& \tau_{l}=\frac{V_{l}}{k_{t} K_{g l} A_{l}} .
\end{aligned}
$$

where $\tau_{l}$ is the characteristic time of the volatile compound depletion in the liquid phase.

The mass flux of aroma released by the source can be expressed as:

$$
\dot{m}\{t\}=j\{t\} A_{l}=k_{t} K_{g l} A_{l} C_{l}\{t=0\} \exp \left(-t / \tau_{l}\right) .
$$

Equation (10) constitutes the aroma release function.

\subsection{Aroma release model for pure volatile compounds}

To facilitate the assessment of the dispersion model, experiments were performed with aroma release from pure liquid compounds. In this case, the release mass flux is constant in time, provided steady air velocity and isothermal conditions are ensured near the source.

If the source contains a pure volatile compound, the release will be governed by the transfer resistance in the gaseous boundary layer. In this case, the mass flux density is still 
given by equation (2), but the interfacial concentration $\left(C_{g}^{*}\right)$ is determined from the saturation vapor pressure of the compound at the gas-liquid interface. With the perfect gas law, the interfacial volatile concentration is given by:

$$
C_{g}^{*}=\frac{M P_{s a t}}{R T}
$$

where $M$ is the molar mass of the compound $(\mathrm{kg} / \mathrm{kmol}), P_{\text {sat }}$ the saturation pressure $(\mathrm{Pa}), R$ the perfect gas constant $(\mathrm{J} / \mathrm{kmol} / \mathrm{K})$ and $T$ the absolute temperature $(\mathrm{K})$.

The mass transfer coefficient in the gas phase $\left(k_{g}\right)$ becomes particularly important for the release flux, as it constitutes the only transfer resistance. It mainly depends on the local stripping air velocity. As in the case of the diluted compound (equation 5), the bulk volatile concentration in the room $\left(C_{g}\right)$ will be usually quite small compared to the interface concentration:

$$
C_{g}<<C_{g}^{*}
$$

This allows decoupling of the release flux calculation by equation (2) from the dispersion calculation of the volatile compound in the room:

$$
j \cong k_{g} C_{g}^{*}
$$

\subsection{Aroma dispersion model}

Aroma dispersion is hereafter discussed in the case of an indoor environment under forced ventilation. An inlet device provides aroma-free air inflow, while an outlet enables the extraction of air containing the aroma compound. Consistently with the assumptions made for computing the mass transfer coefficients, the transport properties of ambient air (i.e. the air density, dynamic viscosity and diffusion coefficients) are assumed to not depend on aroma concentration values. Thus, the availability of a single estimate of the velocity field allows us to study the dispersion of different highly diluted species.

The velocity field can be obtained as the solution of a fluid flow problem under aroma-free conditions. The numerical solution of this problem can be achieved under steadystate conditions. Similarly, the concentration field could be estimated as the solution of the coupled problem of fluid flow and aroma transport under steady-state conditions. However, a number of situations of practical interest involve aroma sources whose release rate cannot be assumed constant with time. In these situations, the dispersion also takes place under transient conditions. 
We first restrict our attention to the evolution with time at a number of selected positions $\vec{x}$ of the mass concentration $C\{\vec{x}, t\}\left(\mathrm{kg} / \mathrm{m}^{3}\right)$ associated with an aroma compound which is released by a constant rate source in an initially aroma-free environment. The solution of the transport problem under transient conditions is reached by considering the velocity field obtained from the aroma-free fluid flow problem. The following boundary conditions are considered: a) aroma is assumed to be released at the source only; b) the aroma mass concentration is set to zero for the incoming air through the inlet device; and c) there are no aroma sinks throughout the computational domain and along its boundaries (room walls, roof and floor). The period of interest starts at the aroma release $(t=0)$ and extends long enough to allow quite steady values for its concentration. The particular situation of a source releasing aroma at constant rate in an initially aroma-free environment can be described in terms of a step function: the mass flux is zero before release starting $(\dot{m}\{t<0\}=0)$ and constant after it $\left(\dot{m}\{t>0\}=\dot{m}_{0}\right)$. Solutions $C\{\vec{x}, t\}$ of the convection-diffusion problem constitute the response of the system to that step release condition.

The transport problem of a given species under transient conditions can be expressed through a convection-diffusion equation:

$$
\frac{\partial C}{\partial t}+\vec{v} \bullet \vec{\nabla} C=\vec{\nabla} \bullet\left(D_{e} \vec{\nabla} C\right)
$$

where $D_{e}$ is the effective diffusivity $\left(\mathrm{m}^{2} / \mathrm{s}\right)$. Acknowledging that this equation is linear in the species concentration, we can define dimensionless quantities and use the convolution theorem for linear dynamic systems. We define dimensionless aroma concentration as follows:

$$
F^{*}\left\{\vec{x}, t^{*}\right\}=C\left\{\vec{x}, t^{*}\right\} / C\left\{\vec{x}_{\text {outlet }}, t^{*}>>1\right\},
$$

where $t^{*}=t / \tau$ is the dimensionless time, $\tau$ is the room renewal time (as usual, defined as the ratio between the room volume and the air inflow rate), and $C\left\{\vec{x}_{\text {outlet }}, t^{*}>>1\right\}$ is the aroma concentration at the room outlet after a period many times longer than the renewal time; the latter is equal to the steady state outlet concentration: $\dot{m}_{0} / \dot{V}$. Dimensionless concentrations $F^{*}\left\{\vec{x}, t^{*}\right\}$ constitute the response to a unit step release condition $\left(\dot{m}\{t>0\} / \dot{m}_{0}=1\right)$. 
More realistic situations involve aroma sources whose release rate cannot to be assumed constant with time after the release starts. Under such situations, the aroma concentration value at given position and time can be estimated from the convolution between the time-dependent mass flux $\dot{m}\{t\}=\dot{m}_{0} f\{t\}$ of aroma compound and the step response function $F^{*}$ associated with the position of interest:

$$
C\{\vec{x}, t *\}=\frac{\dot{m}_{0}}{\dot{V}} \int_{0}^{\infty} \frac{d F^{*}\{\vec{x}, \varsigma\}}{d \varsigma} f\{t *-\varsigma\} d \varsigma .
$$

Figure 1 summarizes the modeling approach here proposed.

Looking for the implementation of this modeling approach:

- The first task is to specify the geometry and the ventilation conditions associated with the indoor environment of interest. The aroma-free, fluid flow problem can hence be represented and then solved under steady-state conditions through a turbulent CFD model; main results are the air velocity and the turbulent intensity fields. Selected results for a given indoor environment are illustrated in subsection 4.1 .

- Input data to be specified include the properties of the considered aroma compounds and the source geometry. The aroma release model detailed in subsection 2.1 is applied to the case of interest; the main result is the mass flux of aroma compound as a function of time.

- The air flow solution can be employed for studying aroma dispersion under different release conditions. In the case of an isothermal inexhaustible source, the steady state aroma concentration depends on the position only. Its spatial distribution can be estimated as the solution of a convection-diffusion problem under steady-state conditions, as illustrated in subsection 4.2 .

- Time-dependent release functions constitute more realistic cases; the aroma concentration depends on position and time too. In the case of constant mass flux after release, the evolution with time of the spatial distribution of the aroma concentration can be estimated as the solution of a convection-diffusion transient problem, assuming aroma-free initial conditions. Such a case is illustrated in subsections 4.3 and 4.4. 
- The previous case of constant mass flux after release can help to study more complicated release conditions. As proposed above, concentration values for the previous case can be interpreted in terms of step responses and later generalized with the help of dimensionless variables. The time evolution of aroma concentration is illustrated in subsection 4.5 for a number of exhaustible sources.

\section{Materials and Methods}

\subsection{Indoor environment}

Figure 2 sketches the main features of the indoor environment under consideration (hereafter, "the room"). It is $7.42 \mathrm{~m}$ long, $6.48 \mathrm{~m}$ wide and $2.92 \mathrm{~m}$ high, with a volume of about $140 \mathrm{~m}^{3}$. Forced ventilation is achieved with an air inlet and an air outlet; both are positioned at the roof. Flow rate of incoming air from outside was stable within $+/-10 \%$ around its nominal value. At the inlet, air crosses a honeycomb panel in order to achieve uniform velocity and low turbulence level. The inlet surface is a square with side $0.40 \mathrm{~m}$; the inlet box is $0.30 \mathrm{~m}$ high. The outlet surface is $0.30 \mathrm{~m} \times 0.20 \mathrm{~m}$. Both the inlet and the outlet are positioned along the longest vertical plane of symmetry of the room.

The case study under consideration includes a table which is also placed at the plane of symmetry. The table is $0.90 \mathrm{~m}$ high and $0.60 \mathrm{~m}$ x $0.60 \mathrm{~m}$ wide, and its sides are closed. The aroma source is placed above the center of the upper side of the table.

\subsection{Measurements}

Measurements of the magnitude of the air velocity and of the aroma concentration have been conducted at a number of selected positions in the indoor environment of interest, under realistic conditions. The experimental effort has been justified by the need of assessing the consistency of numerical simulations of air flow and aroma dispersion; because of our present modeling capabilities, these numerical simulations are feasible under relatively idealized conditions only. Such idealized conditions are presented in next subsection: they include relatively standard assumptions regarding the room geometry and meshing, the fluid flow and the temperature behavior. Under real conditions, some of these assumptions can fail despite our best efforts in reducing, for instance, the importance of obstacles near the walls. Only the comparison of results from repeatable measurements of key variables with those from the model can indicate how much consistent the assumptions are. 
The magnitude of the air velocity has been measured under the air inlet in order to properly evaluate the inlet boundary condition (subsection 3.3), and along vertical and horizontal profiles in order to provide datasets for assessing the consistency of air flow simulations (subsection 4.1). At each position of interest, instantaneous measurements of the air velocity magnitude were performed every $2 \mathrm{~s}$ for a $4 \mathrm{~min}$ period (total of 120 measurements), with the help of a thermoelectric flow sensor (ALMEMO FV-A605-TA) and a data-logger (ALMEMO 2890-9).

Aroma concentration has been measured at selected positions. The aroma source, a Petri dish $13.5 \mathrm{~cm}$ diameter and $2 \mathrm{~cm}$ height containing $100 \mathrm{ml}$ of pure ethyl butyrate, is placed above the center of the upper side of the table. Numbers in Figure 2 indicate the positions of the inlets of eleven sampling lines used for aroma measurements at $1.85 \mathrm{~m}$ above the floor. In the case of the twelfth sampling line, the inlet is positioned inside the air outlet duct, above the roof. The sampling lines are connected to a Proton Transfer Reaction Mass Spectrometry (PTR-MS) analyzer (Ionicon Analytik, Innsbruck, Austria). PTR-MS response was calibrated with the help of gas cylinders containing 100 ppbv of ethyl butyrate in nitrogen. Aroma concentration values were measured every minute. Similar instrumentation has been successfully employed in studying a number of aroma release issues, including the influence of carbonation on the aroma release from selected liquid systems [25] and the volatile release which takes place during eating of natural everyday foods [26].

\subsection{Computational fluid dynamics modeling}

The implementation of our strategy for studying the indoor aroma dispersion requires determination of the air velocity and the turbulent diffusivity fields throughout the domain of interest. As the Reynolds number relative to the air inlet $(\operatorname{Re} \sim 21400$ for an air flow rate of $450 \mathrm{~m}^{3} / \mathrm{h}$ ) is much higher than 2000 , fluid flow cannot be assumed as laminar. That means that velocity fluctuations have to be taken into account. Nevertheless, flow rate is not high enough to ensure full mixing inside the whole room (see results in Section 4). Computational fluid dynamics (CFD) has often been applied as an alternative to experimental research: no restrictions are imposed to the geometry, and relatively high spatial and temporal resolution can be reached regarding flow field and scalar transfer (in our case, concentration).

The flow modeling commercial software ANSYS FLUENT 12 [27] was employed for estimating the air flow field under steady-state and isothermal conditions. The preprocessor software GAMBIT was used for building the computational domain and for generating the 
mesh. The mass conservation equation and the Reynolds-Averaged Navier-Stokes (RANS) equations for three-dimensional fluid flow were the governing equations. The standard kepsilon turbulence model and standard wall functions were used. The equivalent diffusivity $D_{e}$ is almost entirely due to the turbulent (non-laminar) phenomena; it is hereafter estimated by the turbulent diffusivity $D_{t}$. The latter was obtained firstly by computing the turbulent kinematic viscosity as $v_{t}=C_{\mu} k^{2} / \varepsilon$ from the turbulent kinetic energy $k$ and the dissipation rate $\varepsilon$, and then by assuming a turbulent Schmidt number $S c_{t}=v_{t} / D_{t}$ of 0.7 . Therefore, the turbulent diffusivity was directly obtained from the k-epsilon model; no adjustment of $D_{t}$ was undertaken. Continuous governing equations were converted into finite difference equations via integration over control volumes. Main results were obtained after meshing the computational domain into about 2.2 million rectangular hexahedrons; the typical mesh cell was a cube with side $4 \mathrm{~cm}$. Discretization in space was performed through a second-order upwind scheme. Discrete equations were solved under the imposed boundary conditions with an iterative procedure. The modeling software provided a convergent solution after about two days of computation on a 32 Gb RAM memory, 64-bits calculator.

Air inlet velocities were assumed uniform after a number of measurements performed at about 5 centimeters below the air inlet. Figure 3 summarizes the dataset of observed values of air velocity. At a given position, the arithmetic average and the standard deviation values were computed from 120 instantaneous observations performed during periods of 4 minutes. Instantaneous measurements were performed with the help of the abovementioned thermoelectric flow sensor. Positions were distributed across a horizontal rectangular grid, the distance between successive positions being $4 \mathrm{~cm}$. Flow rate of incoming air from outside was stable within $\pm 10 \%$ around the nominal flow rate $450 \mathrm{~m}^{3} / \mathrm{h}$. Values displayed in Figure 3 show the relative homogeneity of air velocities leaving the air inlet.

Selected sensitivity tests were conducted with the air flow model. Figure 4 compares vertical distributions of the air velocity above the table under flow rate $450 \mathrm{~m}^{3} / \mathrm{h}$, firstly obtained after meshing the computational domain with the help of rectangular hexahedrons whose dimensions are close to cubes with side $3,4,6$, and $8 \mathrm{~cm}$ respectively (left hand display), and then obtained by employing two turbulence models (right hand display).

Main results discussed were obtained by meshing the computational domain with the help of about 2.2 million rectangular hexahedrons, close to cubes with side $4 \mathrm{~cm}$. After 
adopting progressively coarser meshes (cubes with sides 6 and then $8 \mathrm{~cm}$ ), the air velocity increases at $1 \mathrm{~m}$ above the table (by about 4 and $6 \%$ ) and decreased in the first centimeters from the roof and the table. Inversely, the adoption of a finer mesh (more than 4 million cubes with side $3 \mathrm{~cm})$ allowed slight changes in the air velocity at $1 \mathrm{~m}$ above the table $(<1 \%)$ and higher air velocities near the roof and the table. The coupled non-linear system of conservation equations for mass, momentum, turbulent kinetic energy and turbulent dissipation rate is known to be more mesh-sensitive than the linear convection-diffusion equation. This is why it was not deemed necessary to conduct a systematic study of the mesh sensitivity for the mass fraction field.

In this study, the standard k-epsilon turbulence model was chosen for obtaining the mean air flow results mainly because its reasonable accuracy for a wide range of turbulent flows. It is the simplest among the turbulent viscosity models: two separate transport equations allow the turbulent velocity and length scales to be solved independently. On the other hand, the Reynolds stress model (RSM) is more elaborate and better takes into account the possible anisotropy of turbulence. The RSM closes the RANS equations by solving transport equations for the Reynolds stresses, together with an equation for the dissipation rate. This means that seven additional transport equations must be solved in three-dimensional problems [27]. In the right hand display of Fig 4, it is shown that these two models do not provide the same air velocity profiles at the center of the room. With respect to RSM, the standard k-epsilon model firstly overestimates the air velocity just above the table (differences below $0.005 \mathrm{~m} / \mathrm{s}$ ), and then underestimates it beyond the first $50 \mathrm{~cm}$ above the table. The differences remain below $0.01 \mathrm{~m} / \mathrm{s}$, so that the simpler k-epsilon model was finally used.

\section{Results}

The following scenario is considered in illustrating the modeling approach: a source of aroma is placed on a table which is located at the center of the room, and the latter is submitted to forced ventilation (inlet and outlet at the roof).

Subsections 4.1 and 4.2 discuss the air velocity, the turbulent diffusivity and the concentration fields obtained under steady-state conditions. Subsections 4.3 and 4.4 discuss the time evolution and the spatial distribution of concentration in the case of constant mass flow after release in an initially aroma-free room. The consistency of selected results is assessed with the help of measurements conducted in the room. Finally, subsection 4.5 
explores the evolution of concentration at selected positions for a time-dependent aroma release.

\subsection{Air flow under steady-state conditions}

The main features of air flow in the room are hereafter discussed with the help of CFD simulations. Fluid properties were held constant with values corresponding to a room temperature of $300 \mathrm{~K}$; air density is $1.225 \mathrm{~kg} / \mathrm{m}^{3}$ and air dynamic viscosity is $1.78910^{-5} \mathrm{~Pa}$.s . Boundary conditions included vanishing air velocities at the walls, at the floor and at the roof. At the air inlet surface, velocity was assumed uniform and normal to the boundary, whereas turbulence was specified by its intensity $(1 \%)$ and by the inlet hydraulic diameter $(0.4 \mathrm{~m})$. Reynolds number was about 21400 under air flow rate $450 \mathrm{~m}^{3} / \mathrm{h}$.

\section{a) Air flow from model}

The main features of the velocity magnitude field are shown in Figure 5 along the plane of symmetry of the room and along the perpendicular plane which crosses the table at the room center. Figure 6 presents selected path-lines starting along these two planes and also along the horizontal plane at $1.85 \mathrm{~m}$ above the floor; path-lines are colored according to the velocity magnitude (note the different scale with respect to Figure 5).

The downward jet incoming through the air inlet drives the air flow field in the room. This jet pushes downward to the ground not only fluid parcels coming from the building's air conditioning system but also fluid parcels already in the room. Such an air intake affects a large portion of the upper part of the room. The downward jet is diverted when approaching the ground; air parcels move towards the walls, where they start upward motion. A threedimensional air flow field can be recognized, which includes circulation patterns between the plane of symmetry and the walls (Figure 6, display A), downward flow along the plane of symmetry (display B), and horizontal flow from the walls to the plane of symmetry at $1.85 \mathrm{~m}$ above the floor (display C).

Turbulent intensity is a major variable for the problem under consideration; it provides the magnitude of turbulent velocity fluctuations relatively to the mean velocity. Figure 7 displays the turbulent intensity along the same planes than in Figure 5. Relevant features are a) the sharp increase from the axis of the downward jet to its outer limits, including the values beyond $100 \%$ in the mixing layer, b) the progressive decrease towards the walls and the center of the room, including a tongue-like flow structure near the floor, and c) relatively low 
values (below $25 \%$ ) over wide regions. We can state that the main source of turbulence in the room is the shear occurring between the downward jet below the air inlet and relatively calm conditions prevailing in its vicinity.

The presence of the table at the room center affects the air flow field; the development of the tongue-like structure near the floor is interrupted (Figure 5), circulation patterns can be identified up- and downstream the table, respectively (Figure 6, display B), and turbulent intensity increases in the central region of the room, relatively to the condition without any table (Figure 7). The presence of the table with the aroma source affects the air flow conditions under which the aroma compound is dispersed.

b) Air velocity from model and experiment

In the scope of this study, the indoor air flow field has been simulated under idealized conditions (simple geometry, isothermal conditions), with the help of a standard turbulence model (subsection 3.3). With the aim of assessing the consistency of air flow simulations under the realistic conditions prevailing in the indoor environment of interest, measurements of the air velocity were conducted at selected positions.

The velocity field in the room was sampled with the help of the same instrument used below the air inlet (subsection 3.3). Figure 8 compares air velocities measured at selected positions and corresponding values retrieved from CFD results, along the plane of symmetry of the room, under the flow rate $450 \mathrm{~m}^{3} / \mathrm{h}$. The air velocity measured at a given position is summarized by the arithmetic average and the standard deviation values computed from 120 instantaneous observations performed during periods of 4 minutes. Measurements were conducted during periods ranging from 2 to 3 hours. Left- and right-hand displays of Figure 8 compare air velocities at $1.85 \mathrm{~m}$ above the floor and at selected heights above the table, respectively.

Apart from the conditions associated with the downward jet below the air inlet (lefthand side of Figure 8), standard deviation values are typically large (30\% or more) when compared to the corresponding mean velocity values. The variation can be partially due to the variability of the incoming air flow rate $( \pm 10 \%)$, to the performance of the thermoelectric sensors employed under turbulent conditions, and to changing flow pattern inside the room.

There is general agreement between air velocities from model and experiment, particularly in the case of the downward jet below the air inlet (left-hand side of Figure 8) and 
of the whole profile above the table (right-hand side of Figure 8). We observe that the level of agreement between air velocities from model and experiment decreases when velocities from model are smaller than $0.05 \mathrm{~m} / \mathrm{s}$. Such a value can be understood as the lower limit of our experimental capabilities.

\subsection{Concentration field under steady-state conditions}

The aroma dispersion in the indoor environment of interest can be firstly studied for the particular case where the mass flux of aroma compound released by the source, $\dot{m}$, is constant with the time, as from an ideally inexhaustible source of aroma. In such a case, the aroma concentration depends on the position only, and its spatial distribution can be estimated as the solution of a convection-diffusion problem under steady-state conditions. At the source, the geometry release corresponds to the experimental setup which effectively has been used in the test room under consideration (a square with side $8 \mathrm{~cm}$, hence $A_{t r}=0.0064 \mathrm{~m}^{2}$ ), and the release velocity is assumed to be an upward velocity of $0.0001 \mathrm{~m} / \mathrm{s}$. Same density as for air is assumed for the aroma compound. At the source, its mass fraction is set to unity.

Figure 9 presents the aroma concentration field along the sample planes as in Figures 5 and 7. A complementary view is offered in Figure 10, including the horizontal plane located at $1.85 \mathrm{~m}$ above the floor. The lowest values occur in the downward jet below the air inlet, as a consequence of the incoming aroma-free boundary condition. The highest concentration values occur in the vicinity of the table; above it, they decrease rapidly as a consequence of the downward air flow pattern. Along the vertical plane which is normal to the plane of symmetry, concentration values exhibit relative maxima (Figure 9); such a feature seems to be associated with circulation patterns prevailing on that plane (Figure 6, display A). Relative maxima can also be identified at $1.85 \mathrm{~m}$ above the floor, along wide regions associated with air flow oriented from the walls towards the plane of symmetry (Figure 10).

In the scenario under consideration, aroma is released at the room center and hence under the influence of downward air flow. Aroma is progressively dispersed along a pathway which includes downward flow towards to the walls, subsequent upward motion and finally air flow towards the plane of symmetry, while approaching the air outlet. The concentration field exhibits a spatial distribution which is far from uniform, as a consequence of the threedimensional air flow field prevailing in the room. 
Figure 10 includes also the positions of the inlets of eleven sampling tubes used for aroma measurements at $1.85 \mathrm{~m}$ above the floor. According to Figure 10, the distribution of such positions appears satisfactory in the sense that the spatial distribution of the concentration field can be sampled in a representative manner.

\subsection{Concentration time evolution under constant release in initially aroma-free room}

A second step in studying the aroma dispersion in the indoor environment of interest assumes that the room is initially aroma-free, that the release starts at time zero, and that the release flux remains constant afterwards. In such a case, the aroma concentration depends not only on the position but also on time, and it can be estimated as the solution of a convectiondiffusion problem under transient conditions. Two air flow rates are considered in this exploratory study, 450 and $900 \mathrm{~m}^{3} / \mathrm{h}$. These flow rates are respectively lower and higher than the typical ventilation rate recommended [28] if the room considered in the study was used as an office, at full occupation. The room renewal time $(\tau)$ associated with each of these conditions can be obtained as usual as the ratio between the room volume and the air flow rate; we obtain $\tau=1120$ and $560 \mathrm{~s}$ under 450 and $900 \mathrm{~m} 3 / \mathrm{h}$ respectively. In studying the time evolution of concentration values, we have limited the period of integration to three hours since the beginning of the aroma release. Such a time period was chosen because it is nearly 10 times larger larger than the room renewal time under the air flow rates of interest.

Figure 11 presents the aroma concentration as a function of time at selected positions, under air flow rates 450 (left) and $900 \mathrm{~m}^{3} / \mathrm{h}$ (right hand displays). The air inlet and outlet devices and also the table with the source are all placed along the longest vertical plane of symmetry of the room; it is hence enough to discuss the concentration evolution for the sampling lines located on this plane (outlet, tubes \#5, \#8, and \#11) and for the remaining positions distributed on the same half-room (tubes \#1, \#6, \#7, and \#12). As shown in Figure 11 , the evolution of concentration values can be different from one position to another during the first minutes after aroma release; further, concentration values increase during a period covering a few renewal times and then exhibit a quite steady pattern. Finally, the range of concentration values obtained after three hours of integration depend on the air flow rate: the aroma mass fraction ranges from 3.2 up to $6.010^{-6}$ under flow rate $450 \mathrm{~m}^{3} / \mathrm{h}$, and from 1.6 up to $3.010^{-6}$ under $900 \mathrm{~m}^{3} / \mathrm{h}$. Such a rough tendency for dividing by two the mass fraction after doubling the flow rate can also be observed at the outlet: $5.110^{-6}$ and $2.610^{-6}$ under 450 and $900 \mathrm{~m}^{3} / \mathrm{h}$ respectively. 
These results are summarized in Figure 12, with the help of the dimensionless concentration defined by equation (15) and the dimensionless time $t^{*}=t / \tau$. Figure 11 shows the outlet and the positions in the room where the aroma concentration is expected to be the lowest and the highest. As shown in this figure, the replacement of absolute scales by dimensionless ones allows the same evolution of concentration with time; at a given position, differences between dimensionless concentration values obtained under flow rates 450 and $900 \mathrm{~m}^{3} / \mathrm{h}$ are typically smaller than $5 \%$. Similar patterns are obtained at each sampling position beyond three renewal times; it corresponds to about the first hour under $450 \mathrm{~m}^{3} / \mathrm{h}$ and the first 30 minutes under $900 \mathrm{~m}^{3} / \mathrm{h}$. We can conclude that, under relatively high Reynolds number at the room inlet (about 21400 under $450 \mathrm{~m}^{3} / \mathrm{h}$ ), similar air flow patterns prevail after doubling the air flow rate (e.g. from 450 to $900 \mathrm{~m}^{3} / \mathrm{h}$ ).

Figure 12 includes for comparison the concentration evolution in the case of a perfectly mixed reactor (white squares), whose dimensionless concentrations are expressed by $1-\exp \left(-t^{*}\right)$. The outlet concentration appears quite close to the concentration in a perfectly mixed reactor beyond $50 \%$ of the first renewal time. Our numerical simulations indicate that the room does not operate as a perfectly mixed reactor because there are positions (for instance tubes \#6 and \#11) where the steady concentration values are significantly higher or lower values than at the outlet. Relative differences with the perfect mixed case are always large at the beginning of the release.

\subsection{Concentration spatial distribution under constant release in initially aroma-free room}

Aroma concentration has been measured in the test room with the aim of assessing the consistency of model results under the realistic conditions. Figure 13 displays an example of a dataset obtained with the table at the room center and under an air flow rate of $900 \mathrm{~m} 3 / \mathrm{h}$. Pure ethyl butyrate in isothermal conditions has been used, allowing a mass flux of aroma compound $(\dot{m})$ constant with time. Three full repetitions are included (black, bold grey and grey symbols); they provide essentially the same information regarding the time evolution of aroma concentration at selected positions.

The availability of concentration measurements over a period of many renewal times can help us to assess the consistency of model results regarding the concentration spatial distribution. The latter can be summarized with the help of time-averaged values at selected positions, computed by including all the instantaneous measurements taken beyond the three first renewal times $(t>3 \tau)$. The interest of such time-averages comes primarily from the 
variability of concentration measurements with time (Figure 13). We argue that results from model and experiment can be suitably compared in terms of dimensionless values, as in Figure 12. Firstly, such normalization allows the comparison of datasets involving different units (mass fractions from model, volume fractions from experiment); secondly, it allows the comparison with a perfectly mixed reactor.

Figure 14 presents dimensionless concentrations from those three repetitions, as a function of the distance from the wall nearby the air inlet. The upper display corresponds to sampling positions which are located $1.5 \mathrm{~m}$ to the left (black symbols) and to the right (white symbols) from the plane of symmetry, while the lower display corresponds to the positions located along the plane of symmetry (Figure 2). Dimensionless concentrations were obtained after evaluating the time-averaged concentration at every sampling position, and then dividing it by the time-averaged concentration at the outlet. All the concentration values observed beyond three renewal times since the aroma release (about $1680 \mathrm{~s}$ in this case) were included in evaluating time-averages and standard deviations; as shown in Figure 12, quite steady conditions for most positions can be reached after such a period.

Numerical simulation of the air flow field was performed by considering a half room only, after assuming that the two halves are identical. Results from experiment allow us to discuss the validity of this assumption; in such a case, practically the same time-averaged concentration values would be obtained for tubes \#10 and \#12 and so forth. Upper display in Figure 14 shows that this is precisely the case for tubes \#7 and \#9, and to a smaller extent for the remaining pairs. Regarding the aroma dispersion under real conditions, the test room does not operate as an idealized symmetrical system with respect to the plane crossing the air inlet and outlet devices. Looking for a possible explanation, temperature measurements at selected positions along the walls were recorded during a week; however, temperature differences were not larger than $1{ }^{\circ} \mathrm{C}$, suggesting little influence on the air flow field.

The hypothesis regarding the operation of the room as a perfectly mixed reactor for aroma dispersion is that it provides a uniform concentration field, whose value equals that at the outlet. In such a case, all the dimensionless concentration values would be centered at unity under steady conditions. Figure 14 shows that this is the case for tube \#11 and to a smaller extent for tubes \#3 and \#4; remaining tubes are associated with progressively larger departures from the perfectly mixed condition. Sampling positions \#10 and \#8 are associated with the minimum and the maximum dimensionless concentrations: 0.71 and 1.11 
respectively, that is $29 \%$ less and $11 \%$ more than the perfectly mixed condition. The local variability of concentration values is indicated by the standard deviations in Figure 14. Sampling position \#10 corresponds to minimum dimensionless concentrations which are significant (standard deviation of about $6 \%$ ); on the other hand, any remark regarding the sampling position \#8 has to be taken with caution because its standard deviation (about $30 \%$ ) can easily mask the departure from the perfectly mixed condition (about $11 \%$ ).

Figure 14 includes dimensionless concentrations from model (crosses). As in the case of concentration values from experiment, only results beyond three renewal times were considered. Dimensionless concentrations from model increase with distance from the wall nearby the air inlet. Such an increase can be explained by the fact that the room is ventilated with aroma-free air, hence the aroma concentration is expected to progressively increase from the inlet to the outlet. Apart from the plane of symmetry (upper display), dimensionless concentrations from model can exhibit values smaller or higher than that at the outlet, depending on the distance from the wall nearby the air inlet.

Figure 14 shows that dimensionless concentrations from model and experiment agree better than 0.15 in the case of sampling positions \#1, \#3, \#5,\#10, and \#12. Air flow conditions around these positions as estimated by CFD can be illustrated by those prevailing at position \#5: air velocity of $0.1-0.15 \mathrm{~m} / \mathrm{s}$ and turbulent intensity of 20-25\% (Figures 5 and 7). On the other hand, differences between dimensionless concentrations from model and experiment reach $0.35-0.40$ at positions $\# 8$ and \#11. Air flow conditions around these two positions are not very different from those prevailing at position \#5; actually, the turbulent intensity is somewhat higher at position \#11 (about 25-30\%, Figure 7).

Numerical simulations of the air flow field were obtained by employing the RANS equations; they provided useful results regarding the mean air flow behavior as well as the effects of fully developed small scale turbulence on the mean air flow properties. However, under realistic conditions, turbulent flow can be associated with spatial scales which are not resolved with the help of the RANS modeling approach.

In the scope of this study, it seems plausible to associate turbulent intensity with the variability of concentration with time. The latter can be assessed by the standard deviations shown in Figure 14; for a given position, they were evaluated from all the available concentration observations beyond three renewal times, by taking into account the three repetitions. Such standard deviations have values of about 0.18 and 0.30 at positions \#11 and 
\#8, where the differences between dimensionless concentrations from model and experiment reach 0.35-0.40. Sampling positions \#11 and \#8 were associated with the highest concentration variability with time as revealed by experiment (Figure 13). On the other hand, the standard deviation is smaller than 0.05 at position $\# 5$, where dimensionless concentrations from model and experiment agree better than 0.15. It appears that time-averaged concentration values from model and experiment almost agree when the variability of concentration values with time is relatively low $(<10 \%)$. In the region of high variability, we can conjecture that the large turbulent structures which are not captured by the RANS approach should be taken into account to improve the prediction.

The spatial distribution of aroma concentration in the room at a height of $1.85 \mathrm{~m}$ is characterized by a first increase tendency from the air inlet to the outlet and by a second increase tendency from the plane of symmetry towards the walls. Both tendencies seem to be stronger from model than from experiment. Further, model results exhibit a larger departure, than those from experiment, with respect to the perfectly mixed reactor behavior.

\subsection{Concentration time evolution under time-dependent release in initially aroma-free room}

Previous subsections have discussed the time evolution and the spatial distribution of aroma concentration under constant release. Many scenarios of practical interest involve aroma sources whose release mass flux cannot be assumed constant with time, as in the case of diluted solutions. Such a time-dependent release rate can affect both the time evolution and the spatial distribution of the aroma concentration; the latter can be estimated by solving the partial derivative equations which describes the convection-diffusion problem of interest. The expensive task of solving this challenging problem can be avoided with the help of the modeling approach presented in Section 2. Firstly, aroma release and aroma dispersion have been decoupled: ambient air transport properties were assumed do not depend on aroma concentration, which in turn was assumed to be far from its "saturation level" in the room. Secondly, the indoor air velocity and turbulence intensity fields have been estimated under steady-state conditions, hence no additional CFD simulations are required. Thirdly, the time evolution of aroma concentration after release starting has been obtained under constant release and later interpreted in terms of step responses. Finally, the aroma concentration as a function of position and time can be estimated by convolving such responses with the timedependent mass flux which characterizes the scenario of interest (equation 16). 
The mass flux of aroma compound released by a source when the aroma is in solution, $\dot{m}$, has been defined (equation 10) as a function of the cross-sectional release area $A_{l}$, the total mass transfer coefficient $k_{t}$, the gas-liquid partition coefficient $K_{g l}$, the initial aroma concentration $C_{l}\{t=0\}$ in the liquid source, and the volume $V_{l}$ associated with the liquid source. Some of these variables play a combined role in the case of the release decreasing characteristic time, $\tau_{l}$ (equation 9). Different scenarios can be associated with a same characteristic time, hence providing a same time evolution for the aroma concentration in the liquid source, and finally giving a same dispersion pattern throughout the room. The potential of our modeling strategy can be put in evidence by choosing selected scenarios, each of them associated with a given value for the release decreasing characteristic time, rather than by electing particular values for every key variable abovementioned.

Dimensionless concentrations are hereafter compared under a variety of scenarios at one selected position only. Figure 15 summarizes results obtained under the flow rate 900 $\mathrm{m}^{3} / \mathrm{h}$, after assuming four different aroma sources. Similarly to Figure 12 , dimensionless concentrations are presented as a function of the dimensionless times. The thick black curve corresponds to an inexhaustible source; for such a source, dimensionless concentrations reach a quite steady value after three renewal times.

The three remaining curves in Figure 15 were obtained for three sources associated with different values of the release decreasing characteristic time $\tau_{l}$; for convenience, they were expressed in terms of the room renewal time $\tau$. Such a representation allows a broader analysis regarding the relative roles of these two characteristic times. White squares in Figure 15 correspond to a source with a relatively high value for $\tau_{l}$ (100 times $\left.\tau\right)$; the aroma mass flux slowly decreases with time, representing a scenario associated with relatively long-term aroma release when compared with the air renewal in the room. As a consequence, the time evolution of dimensionless concentration initially follows that for an inexhaustible source (roughly, up to two renewal times) and then slowly decreases. Grey and black squares in Figure 15 correspond to situations in which $\tau_{l}$ is firstly twice $\tau$ and then half of that value, respectively. In both cases the time evolution of dimensionless concentration starts following the previous situation, then exhibits a maximum value, and finally vanishes beyond a few renewal times. The maximum value is weaker, and the time interval before vanishing is shorter, when the renewal time is higher than the release decreasing characteristic time. 
Results presented in Figure 15 demonstrate the potential of our modeling strategy. It is sufficiently flexible for representing the indoor aroma dispersion following its release from either inexhaustible or time-dependent sources.

\section{Conclusions}

In this article we have proposed and tested a novel mechanistic approach for studying the dispersion of aroma under indoor conditions. The approach combines a computational fluid dynamics (CFD) model which predicts air flow and scalar convection-diffusion of species, with an aroma release model.

The major advantage of our modeling approach is its flexibility: air flow field was computed separately from aroma release and dispersion. Hence, different release models as well as the release of distinct aroma compounds can be directly studied by taking into account a same velocity field, without any additional CFD simulation. The ANSYS FLUENT 12 flow modeling simulation software was employed for estimating the mean air flow field and for solving the convection-diffusion problem under transient conditions for a constant release rate, while the aroma release model and the convolution (equation 15) were developed in the programming language MATLAB. The application of our modeling approach to other software and programming language seems feasible.

The consistency of the mean air flow from model was assessed from the analysis of measurements of the air velocity along a vertical line at the room center and along a horizontal line at $1.85 \mathrm{~m}$ above the floor. Differences between air velocities from model and experiment grew when the velocities became relatively weak (smaller than $0.05 \mathrm{~m} / \mathrm{s}$ ).

Dimensionless variables were introduced as a tool for studying the indoor aroma dispersion: the concentration at a given position is normalized to the steady value at the room outlet, whereas the time is divided by the room renewal time. Results from model indicated that dimensionless concentrations at a given position can exhibit essentially the same evolution with dimensionless time after doubling the air inflow rate.

Results from the modeling approach were compared to measurements of aroma compound volume fraction at selected positions in the case of an inexhaustible source. Dimensionless concentrations from model and experiment were compared after time- 
averaging beyond the first three renewal times. Time-averaged concentration values from model and experiment agree better under conditions associated with relatively small turbulent intensity (small concentration variations with time). Under realistic conditions, turbulent flow can be associated with large transient structures which are not resolved with the help of the RANS modeling approach. The knowledge built from CFD results here discussed may not be sufficient for completely understanding aroma dispersion in the room. A more complete study of indoor air flow behavior should involve the large-eddy simulation (LES) approach, but requiring computer memory and speed beyond our present capabilities.

In summary, both the mean air flow pattern and the aroma convection are reasonably well predicted with the help of our modeling approach. Next efforts include the application of this approach to a broader set of conditions.

\section{Acknowledgements}

Authors thank Mireille Moser (Nestlé Research Center, Switzerland), Isabelle Deleris and Steven Le Feunteun (Institut National de la Recherche Agronomique, France), and Jean Moureh (Institut National de Recherche en Sciences et Technologies pour l'Environment et l'Agriculture, France) for helpful discussions along this research. Acknowledgements are also addressed to W. W. Nazaroff (University of Berkeley, California) for helpful suggestions.

\section{References}

1. R. S. T. Linforth, E. N. Friel, A. J. Taylor, in Flavor Release: Linking Experiments, Theory and Reality (Eds.: D. D. Roberts, A. J. Taylor), American Chemical Society Symposium Series 763, 2000, pp. 166-178.

\section{A. J. Taylor, Compr. Rev. Food Sci. Food Saf. 2002, 1, 45.}

3. R. S. T. Linforth, in Food Flavour Technology (Eds.: A. J. Taylor, R. S. T. Linforth), 2nd edition, Wiley-Blackwell, 2010, pp. 207-228.

4. van Ruth, S. M., J. P. Roozen, in Food Flavour Technology (Eds.: A. J. Taylor, R. S. T. Linforth), 2nd edition, Wiley-Blackwell, 2010, pp. pp. 190-206.

5. I. Gierczynski, E. Guichard, H. Laboure, Flavour Fragr. J. 2011, 26, 141. 
6. I. C. Trelea, S. Atlan, I. Deleris, A. Saint-Eve, M. Marin, I. Souchon, Chem. Senses 2008, 33,181 .

7. L. W. Turley, R. E. Milliman, R. E., J. Busn. Res. 2000, 49, 193.

8. A. J. Gadgil, C. Lobscheid, M. O. Abadie, E. U. Finlayson, Atmos. Environ. 2003, 37, 5577.

9. J. H. Seinfeld, S. N. Pandis, Atmospheric Chemistry and Physics: from Air Pollution to Climate Change, John Wiley and Sons Inc., New York (USA), 1998.

10. F. Pasquill, Q. J. R. Meteorolog. Soc. 1971, 97, 369.

11. N. S. Holmes, L. Morawska, Atmos. Environ. 2006, 40, 5902.

12. J. D. Posner, C. R. Buchanan, D. Dunn-Rankin, D. Energy Build. 2003, 35, 515.

13. Z. Zhang, Q. Chen, Atmos. Environ. 2006, 40, 3396.

14. H. Sun, L. Zhao, Y. Zhang, Tran. Am. Soc. Agric. Biol. Eng. 2007, 50, 621.

15. S. B. Poussou, S. Mazumdar, M. W. Plesniak, P. E. Sojka, Q. Chen, Atmos. Environ. 2010, 44, 2830.

16. Y. Li, P. V. Nielsen, Indoor Air 2011, 21, 442.

17. X. Yang, Q. Chen, J. S. Zhang, R. Magee, J. Zeng, C. Y. Shaw, Build. Environ. 2001, 36, 1099.

18. R. L. Corsi, C.-C. Lin, Crit. Rev. Env. Sci. Technol. 2009, 39, 1052.

19. European Commission, Evaluation of VOC Emissions from Building Products, European Collaborative Action "Indoor Air Quality and Its Impact on Man" (report No 18), European Commission, Luxembourg, publication EUR 17334, 1997.

20. Z. Guo, Environ. Pollut. 2002a, 120, 533.

21. Z. Guo, Environ. Pollut. 2002b, 120, 551.

22. Z. Liu, W. Ye, J. C. Little, Build. Environ. 2013, 64, 7.

23. M. Marin, I. Baek, A. J. Taylor, J. Agric. Food Chem. 1999, 47, 4750. 
24. E. L. Cussler, Diffusion: Mass Transfer in Fluid Systems, Cambridge Univ. Press, Cambridge (UK), 1984.

25. M. A. Pozo-Bayon, M. Santos, P. J. Martin-Alvarez, G. Reineccius, Flavour Fragr. J. 2009, 24, 226.

26. D. C. Frank, G. T. Eyres, U. Piyasiri, C. M. Delahunty, Flavour Fragr. J. 2012, 27, 433.

27. ANSYS, ANSYS FLUENT 12.0 - Theory Guide, ANSYS Inc., Pennsylvania (USA), 2009.

28. J. Sundell, H. Levin, W. W. Nazaroff, W. S. Cain, W. J. Fisk, D. T. Grimsrud, F. Gyntelberg, Y. Li, A. K. Persily, A. C. Pickering, J. M. Samet, J. D. Spengler, S. T. Taylor, C. J. Weschler, Indoor Air 2001, 21, 191. 
We propose a modeling approach for studying the indoor dispersion of aroma compounds which are released from, for instance, food products. We show that the indoor velocity field can be estimated separately through CFD, under aroma-free conditions, and then combined with the aroma mass flow associated with the source of interest. Modeling results compare favorably with experimental measurements. The modeling approach is flexible and computationally effective, since different release conditions can be considered with no additional CFD simulation. $270 \times 203 \mathrm{~mm}(96 \times 96$ DPI) 


\section{INPUT DATA}

Figure 1: Schematic representation of the modeling approach. $1587 \times 1190 \mathrm{~mm}(96 \times 96 \mathrm{DPI})$ 
Figure 2: Main features of the indoor environment under consideration. Air inlet and outlet devices are indicated, as well as the source of aroma above a table at the room center. The locations of the twelve sampling lines used for concentration measurements are also indicated. Sampling positions $1,3,4 \ldots 12$ are located along the horizontal plane at $1.85 \mathrm{~m}$ above the floor, and sampling position 2 is located at the air outlet.

$270 \times 203 \mathrm{~mm}(96 \times 96$ DPI $)$ 
Figure 3: Observed values of the air velocity $(\mathrm{m} / \mathrm{s})$ at selected positions about 5 centimeters below the air inlet, under nominal flow rate $450 \mathrm{~m} 3 / \mathrm{h}$. Observations at a given position are summarized by the arithmetic average and the standard deviation values (in parenthesis) computed from 120 instantaneous observations. The inlet surface is a square with side $0.40 \mathrm{~m}$; it is here displayed as seen by an observer at the ground, looking upwards. $1587 \times 1190 \mathrm{~mm}(96 \times 96 \mathrm{DPI})$ 
Figure 4: Air velocities from the model at selected positions above the table, under flow rate $450 \mathrm{~m} 3 / \mathrm{h}$. Left hand display compares results obtained after meshing the computational domain into rectangular hexahedrons whose dimensions are close to those of cubes with side $3,4,6$, and $8 \mathrm{~cm}$ respectively; the standard k-epsilon turbulence model is assumed. Right hand display compares results obtained after assuming the RSM and the standard k-epsilon turbulence models; the mesh is constituted of rectangular hexahedrons close to cubes with side $4 \mathrm{~cm}$. $1587 \times 1190 \mathrm{~mm}(96 \times 96 \mathrm{DPI})$ 
Figure 5: Air velocities from model along two vertical planes under the flow rate $450 \mathrm{~m} 3 / \mathrm{h}$. $270 \times 203 \mathrm{~mm}(96 \times 96$ DPI $)$ 

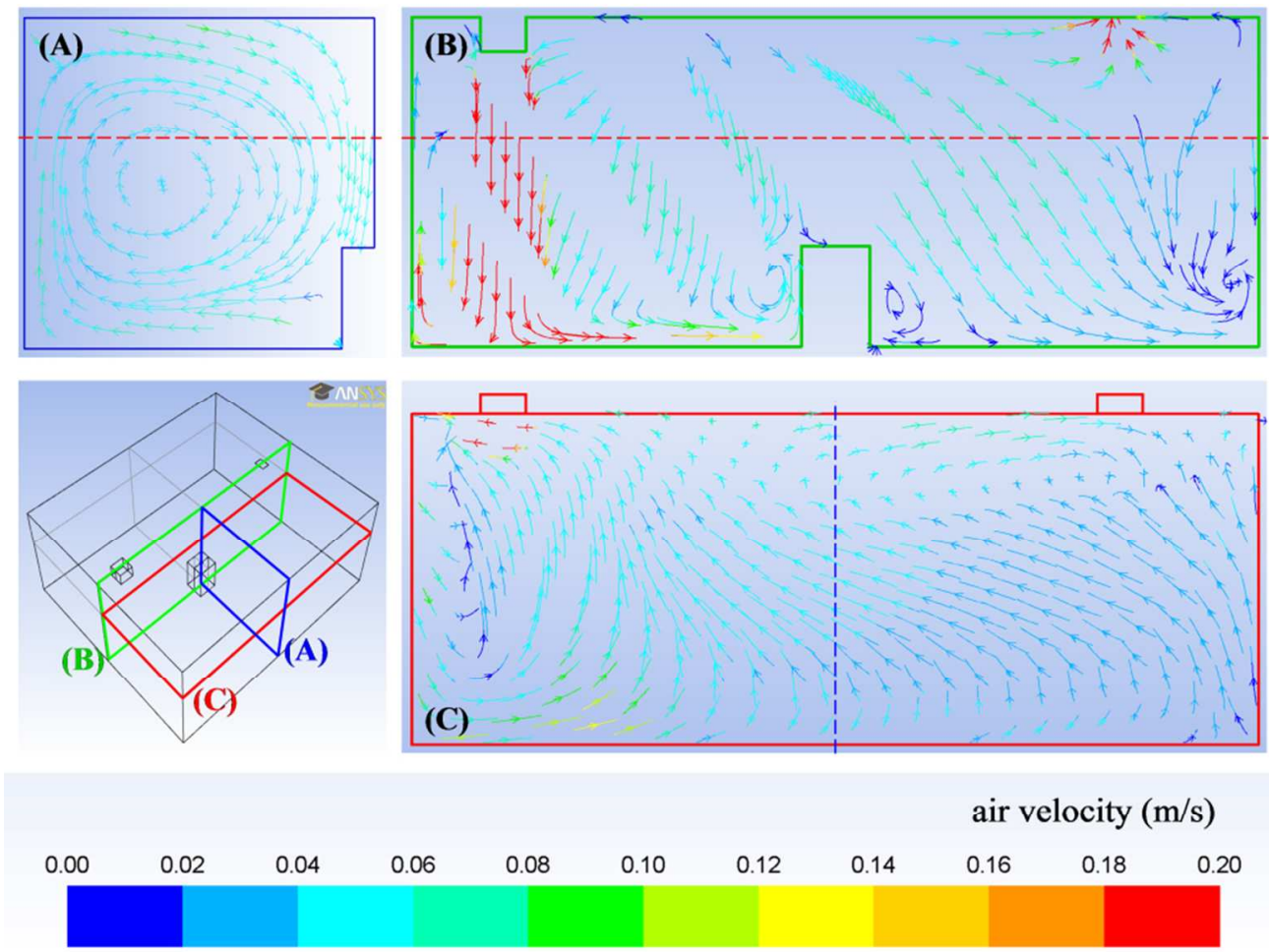

Figure 6: Path-lines from the model along selected planes under the flow rate $450 \mathrm{~m} 3 / \mathrm{h}$ : A) along the vertical plane crossing the plane of symmetry at the room center, B) along the plane of symmetry, and C) at $1.85 \mathrm{~m}$ above the floor for a downward-looking observer at the roof. The locations of the air inlet and outlet are indicated in planes $B$ and $C$. The height associated with the plane $C$ is indicated by the red dashed line in planes $A$ and $B$. The ordinate associated with the plane $A$ is indicated by the blue dashed line in plane $C$. The lower left hand display summarizes the relative position of the three planes under consideration. Path-lines are colored according to the velocity magnitude; red path-lines may indicate air velocities above $0.2 \mathrm{~m} / \mathrm{s}$. $270 \times 203 \mathrm{~mm}(96 \times 96$ DPI $)$ 
Figure 7: Turbulent intensity from model along two vertical planes under the flow rate $450 \mathrm{~m} 3 / \mathrm{h}$. Values above $100 \%$ occur below the air inlet and nearby the outlet; they are omitted for the sake of clarity. The location of sampling positions \#5, \#8, and \#11 is also indicated. $270 \times 203 \mathrm{~mm}(96 \times 96$ DPI $)$ 
Figure 8: Air velocities from model and experiment at selected positions, under nominal flow rate $450 \mathrm{~m} 3 / \mathrm{h}$. Left-hand display presents results at positions which are located $1.85 \mathrm{~m}$ above the floor, along the plane of symmetry; distances are measured from the wall nearby the air inlet. Right-hand display presents results above the table, at the room center; distances are measured from upper face of the table. Velocities from model were sampled at selected distances from CFD results. Velocities from experiment are summarized by the arithmetic average and the standard deviation values from 120 instantaneous measurements. $1587 \times 1190 \mathrm{~mm}$ (96 × 96 DPI) 
Figure 9: Aroma mass fraction from model along two vertical planes under the flow rate $450 \mathrm{~m} 3 / \mathrm{h}$. Values near the source range from 10-5 up to the unity. Selected path-lines shown in Figure 6 (displays A and B) are also indicated; air velocities are not in scale. $270 \times 203 \mathrm{~mm}$ (96 x 96 DPI) 
Figure 10: As in Figure 9, but including results along the horizontal plane at $1.85 \mathrm{~m}$ above the floor. The location of the sampling positions is indicated. Selected path-lines shown in Figure 6 (display C) are sketched; air velocities are not in scale. $270 \times 203 \mathrm{~mm}(96 \times 96 \mathrm{DPI})$ 
Figure 11: Aroma mass fraction values from model as a function of time since beginning of release at selected positions under the flow rate 450 (left displays) and $900 \mathrm{~m} 3 / \mathrm{h}$ (right displays). Outlet excepting, all the positions are located $1.85 \mathrm{~m}$ above the floor. Top displays present the whole period of three hours of integration, while the bottom displays focus on the first 5 minutes. $1587 \times 1190 \mathrm{~mm}(96 \times 96 \mathrm{DPI})$ 
Figure 12: Dimensionless concentrations from model as a function of the dimensionless time at selected positions under the flow rate 450 (grey curves) and $900 \mathrm{m3} / \mathrm{h}$ (black curves). Outlet excepting, all the positions are located $1.85 \mathrm{~m}$ above the floor. Dimensionless concentrations for a perfectly mixed reactor are also indicated (white squares). Note the logarithmic time scale. $1587 \times 1190 \mathrm{~mm}(96 \times 96 \mathrm{DPI})$ 
Figure 13: Volume fractions in part per billion ( $\mathrm{ppb}$ ) of ethyl butyrate from experiment as a function of time since release at selected positions, under the nominal flow rate $900 \mathrm{~m} 3 / \mathrm{h}$. Outlet excepting, all the positions are located $1.85 \mathrm{~m}$ above the floor. Three replicate experiments are indicated with black, bold grey and grey symbols.

$1587 \times 1190 \mathrm{~mm}(96 \times 96 \mathrm{DPI})$ 
Figure 14: Time-averaged concentration values from model and experiment at selected positions, under the nominal flow rate $900 \mathrm{~m} 3 / \mathrm{h}$. Results from experiment are summarized by arithmetic averages and standard deviations computed over the whole dataset constituted after three repetitions. The upper display shows results along two series of sampling positions located at the same distance from the plane of symmetry. The lower display shows results corresponding to the sampling positions located on the plane of symmetry. Results are presented as function of the distance measured from the wall nearby the air inlet; approximate locations of the inlet and of the outlet are indicated. Dimensionless concentration values in a perfectly mixed reactor correspond to unity (horizontal grey line). $1587 \times 1190 \mathrm{~mm}(96 \times 96 \mathrm{DPI})$ 
Figure 15: Dimensionless concentrations from model as a function of dimensionless time at sampling position \#6 under the flow rate $900 \mathrm{~m} 3 / \mathrm{h}$. Black curve has been displayed in Figure 13, corresponding to results obtained for an inexhaustible source. White, grey and blacks squares correspond to results obtained for hypothetical sources associated with different values for the release decreasing characteristic time (T), namely equal to 100 , twice and half of the room renewal time (T_ell) respectively. $1587 \times 1190 \mathrm{~mm}(96 \times 96 \mathrm{DPI})$ 